\title{
Prevalência e localização espacial dos casos de tracoma detectados em escolares de Botucatu, São Paulo - Brasil
}

\author{
Prevalence and spatial distribution of trachoma among schoolchildren \\ in Botucatu, São Paulo - Brazil
}

Silvana Artioli Schellini ${ }^{1}$, Marcelo Mendes Lavezzo² ${ }^{2}$ Lucieni Barbarini Ferraz ${ }^{3}$, Jaime Olbrich Neto ${ }^{4}$, Norma Hellen Medina ${ }^{5}$, Carlos Roberto Padovani ${ }^{6}$

\section{RESUMO}

Objetivo: Avaliar a prevalência de tracoma em escolares de Botucatu/SPBrasil e a distribuição espacial dos casos.

Métodos: Foi realizado um estudo transversal, em crianças de 7-14 anos, que frequentavam as escolas do ensino fundamental de Botucatu/SP, em novembro/2005. O tamanho da amostra foi estimado em 2.092 crianças, considerando-se a prevalência histórica de 11,2\%, aceitando-se erro de estimação de 10\% e nível de confiança de $95 \%$. A amostra foi probabilística, ponderada e acrescida de 20\%, devido à possível ocorrência de perdas. Examinaram-se 2.692 crianças. O diagnóstico foi clínico, baseado na normatização da Organização Mundial da Saúde (OMS). Para avaliação dos dados espaciais, utilizou-se o programa CartaLinx (v1.2), sendo os setores de demanda escolar digitalizados de acordo com as divisões do planejamento da Secretaria de Educação. Os dados foram analisados estatisticamente, sendo a análise da estrutura espacial dos eventos calculadas usando o programa Geoda.

Resultados: A prevalência de tracoma nos escolares de Botucatu foi de 2,9\%, tendo sido detectados casos de tracoma folicular. A análise exploratória espacial não permitiu rejeitar a hipótese nula de aleatoriedade $(l=-0,45, p>0,05)$, não havendo setores de demanda significativos. A análise feita para os polígonos de Thiessen também mostrou que o padrão global foi aleatório $(\mid=-0,07 ; p=0,49)$. Entretanto, os indicadores locais apontaram um agrupamento do tipo baixo-baixo para um polígono ao norte da área urbana.

Conclusão: A prevalência de tracoma em escolares de Botucatu foi de 2,9\%. A análise da distribuição espacial não revelou áreas de maior aglomeração de casos. Embora o padrão global da doença não reproduza as condições socioeconômicas da população, a prevalência mais baixa do tracoma foi encontrada em setores de menor vulnerabilidade social.

Descritores: Tracoma/epidemiologia; Distribuição espacial da população; Cegueira/prevenção \& controle

\begin{abstract}
Purpose: To assess the prevalence of trachoma in schoolchildren of Botucatu/ SP-Brazil and its spatial distribution.

Methods: Cross-sectional study in children aged from 7 to 14 years, who attended elementary schools in Botucatu/SP in November/2005. The sample size was estimated in 2,092 children, considering the 11.2\% historic prevalence of trachoma, accepting an estimation error of $10 \%$ and confidence level of $95 \%$ The sample was random, weighted and increased by $20 \%$, because of the possible occurrence of losses. The total number of children examined was 2,692. The diagnosis was clinical, based on WHO guidelines. For the evaluation of spatial data, the CartaLinx program (v1.2) was used, and the school demand sectors digitized according to the planning divisions of the Department of Education. The data were statistically analyzed, and the analysis of the spatial structure of events calculated using the Geode program.

Results: The prevalence of trachoma in schoolchildren of Botucatu was $2.9 \%$ and there were cases of follicular trachoma. The exploratory spatial analysis failed to reject the null hypothesis of randomness $(R=-0.45, p>0.05)$, with no significant demand sectors. The analysis for the Thiessen polygons also showed that the overall pattern was random $(l=-0.07, p=0.49)$. However, local indicators pointed to a group of low-low type for a polygon to the north of the urban area.

Conclusion: The prevalence of trachoma in schoolchildren in Botucatu was 2.9\%. The analysis of the spatial distribution did not reveal areas of greater clustering of cases. Although the overall pattern of the disease does not reproduce the socio-economic conditions of the population, the lower prevalence of trachoma was found in areas of lower social vulnerability.
\end{abstract}

Keywords: Trachoma/epidemiology; Residencefeatures; Blindness/prevention \&control
Trabalho realizado junto ao Departamento de Oftalmologia, Otorrinolaringologia e Cirurgia de Cabeça e Pescoço, Faculdade de Medicina de Botucatu, Universidade Estadual Paulista "Júlio de Mesquita Filho" - UNESP - Botucatu (SP), Brasil.

${ }^{1}$ Médica, Departamento de Oftalmologia, Otorrinolaringologia e Cirurgia de Cabeça e Pescoço, Faculdade de Medicina de Botucatu, Universidade Estadual Paulista "Júlio de Mesquita Filho" (UNESP) - Botucatu (SP), Brasil.

2 Médico, Clínica Oftalmológica, Faculdade de Medicina, Universidade de São Paulo - USP - São Paulo - (SP), Brasil.

${ }^{3}$ Médica, Serviço de Oftalmologia, Hospital Estadual Bauru - Bauru (SP), Brasil.

${ }^{4}$ Médico, Departamento de Pediatria, Faculdade de Medicina de Botucatu, Universidade "Estadual Paulista "Júlio de Mesquita Filho" - UNESP - Botucatu (SP), Brasil.

Estadual Paulista "Júlio de Mesquita Filho" - UNESP - Botucatu (SP), Brasil.
${ }^{5}$ Médica, Centro de Oftalmologia Sanitária, Centro de Vigilância Epidemiológica "Prof. Ale5 Médica, Centro de Oftalmologia Sanitária, Centro
xandre Vranjac" (CVE/SES) - São Paulo (SP), Brasil.

Estatístico, Instituto de Biociências, Departamento de Bioestatística, Universidade Estadual Paulista "Júlio de Mesquita Filho" - UNESP - Botucatu (SP), Brasil.

Endereço para correspondência: Silvana Artioli Schellini. Dep. OFT/ORL/CCP - Faculdade de Medicina de Botucatu - UNESP - Botucatu (SP) - CEP 18618-000

E-mail: sartioli@fmb.unesp.br

Recebido para publicação em 31.01.2010

Última versão recebida em 13.07.2010

Aprovação em 22.07.2010

\section{INTRODUÇÃO}

0 tracoma é uma ceratoconjuntivite crônica e recidivante causada pela Chlamydia trachomatis. Afeta crianças desde os primeiros meses de vida, evoluindo para: cicatrização conjuntival, entrópio, triquíase, opacidade corneana, olho seco e cegueira. Geralmente, sua transmissão ocorre dentro do ambiente doméstico, de forma direta (mãos contaminadas) ou indiretamente (vestuários) $)^{(1-2)}$.

É a doença ocular mais frequente do mundo, acometendo 500 milhões de indivíduos ${ }^{(3-4)}$. A Organização Mundial da Saúde (OMS) estima que 5,6 milhões de pessoas estejam cegas devido às complicações do tracoma, sendo, portanto, a principal causa de cegueira prevenível, principalmente nos países em desenvolvimento(5). Além disso, 80 milhões de crianças apresentam o tracoma inflamatório que pode causar grave deficiência visual(6).

Trata-se de uma doença que está associada a fatores socioeconômicos indicativos de alta vulnerabilidade social, condição encontrada, em geral, nas periferias das cidades. 
Em Botucatu/SP, não foram notificados casos de tracoma folicular ou inflamatório nos últimos anos, não obstante ao inquérito prévio, realizado em 1995, ter diagnosticado tracoma em 11,9\% das crianças que frequentavam as escolas ${ }^{(7)}$. Desde então, nenhum tipo de inquérito ou ação de vigilância epidemiológica foram realizados com o intuito de se detectarem casos novos.

O estudo da distribuição espacial do tracoma permitiria verificar se a organização do espaço socioeconômico se reproduz na dinâmica de contágio da doença entre os escolares.

O objetivo do presente estudo foi avaliar a prevalência de tracoma em escolares de 7-14 anos de idade de Botucatu/SP e verificar, usando o método do geoprocessamento, a existência de fatores locais que possibilitassem diferenças na distribuição espacial dos casos detectados.

\section{MÉTODOS}

\section{ANÁLISE dOS dADOS CLÍNICOS}

Realizou-se inquérito epidemiológico em crianças da primeira a quarta séries do ensino fundamental de todas as escolas públicas de Botucatu/SP, em novembro/2005. Foram excluídas as crianças fora da faixa etária escolhida e aquelas cujos responsáveis não autorizaram a participação.

Em 2005, 6.408 alunos frequentavam o ensino fundamental, segundo dados da Secretaria de Ensino de Botucatu.

O tamanho da amostra foi estimado em 2.092 crianças, considerando-se a prevalência de tracoma de 11,2\% (baseada no levantamento anterior de 1995)(7), aceitando-se erro de estimação de $10 \%$ e nível de confiança de $95 \%$. A amostra foi acrescida de $20 \%$, devido à possível ocorrência de perdas ${ }^{(8)}$. As crianças foram selecionadas por amostragem probabilística em conglomerados. Considerou-se, como unidade primária de amostra as classes, tomando-se, como número médio, 30 crianças por sala de aula. As classes foram escolhidas por sorteio, realizado para que se obtivesse uma amostra ponderada, baseada no número de escolas e no número de alunos. Assim, examinaram-se 2.692 crianças.

Todos os indivíduos foram avaliados, sempre pelo mesmo examinador, que realizou o exame oftalmológico usando lupa de 2,5 vezes de magnificação, sob iluminação natural ou artificial, com auxílio de uma lanterna. O método de avaliação seguiu padronização para a identificação do tracoma, segundo critérios da OMS, avaliando-se alterações das pálpebras, cílios, conjuntiva e córnea. Desse modo, o diagnóstico foi clínico, baseado na normatização da OMS para detecção de tracoma ${ }^{(3)}$.

A avaliação dos casos foi realizada utilizando-se técnicas de estatística descritiva e o teste do qui-quadrado para determinação de associações entre variáveis categóricas. Na determinação da amostra, houve correção pelo fato de a amostragem ter sido feita por conglomerados "clustering".

\section{ANÁLISE dOS DADOS ESPACIAIS}

Foram avaliados os padrões global e local de associação espacial para o Índice Paulista de Vulnerabilidade Social (IPVS), calculado pelo Instituto Brasileiro de Geografia e Estatística (IBGE), de acordo com os setores censitários de 2000, e para a prevalência do tracoma, de acordo com o levantamento epidemiológico de $2005^{(7)}$. A abordagem proposta foi realizada no sentido de se estimar a prevalência de acordo com a localização da escola, com o setor de demanda escolar definido pela Secretaria da Educação e com o endereço de residência dos alunos detectados com a doença.

Utilizou-se o programa CartaLinx (v1.2) para converter os dados analógicos em digitais. Os setores de demanda escolar foram digitalizados de acordo com as divisões do planejamento da Secretaria de Educação.

A estimativa da prevalência de tracoma entre os escolares de até 10 anos de idade foi calculada por escola e por setor de demanda escolar. Os dados pontuais referentes às localizações das escolas foram convertidos em polígonos de Thiessen. Dessa forma, foram localizados, no mapa digital, os endereços de todos os alunos detectados como sendo portadores de tracoma.

\section{ANÁlISE EXPLORATÓRIA DOS DADOS ESPACIAIS}

O coeficiente I de Moran mede a probabilidade de um padrão espacial aparente ter sido produzido meramente por acaso e é similar ao coeficiente da correlação de Pearson. Na autocorrelação espacial, a correlação é examinada entre diferentes valores dentro da mesma variável e uma matriz de ponderação é incluída para definir as relações espaciais entre áreas. O coeficiente I é calculado como a razão do produto do desvio médio e as somas dos quadrados das coordenadas $x$ (longitude) e y (latitude):

$$
\mathrm{I}=\frac{\mathrm{N} \sum_{\mathrm{i}} \sum_{\mathrm{j}} \mathrm{W}_{\mathrm{ij}}\left(\mathrm{X}_{\mathrm{i}}-\overline{\mathrm{X}}\right)\left(\mathrm{X}_{\mathrm{j}}-\overline{\mathrm{X}}\right)}{\mathrm{J} \sum_{\mathrm{i}} \sum_{\mathrm{j}} \mathrm{W}_{\mathrm{ij}} \sum_{\mathrm{i}}\left(\mathrm{X}_{\mathrm{i}}-\overline{\mathrm{X}}\right)^{2}}
$$

Onde: $N=$ número de setores censitários no estudo; $J=$ número de pares de setores examinados; $X=$ valor ( $p$. ex. prevalência) do setor $i ; X_{i}=$ valor do setor $j\left(X_{i}\right.$ e $X$ são setores vizinhos) e $W_{\mathrm{il}}=$ matriz de ponderação. $\mathrm{O}$ coeficiente I de Moran varia de $-1 \mathrm{a}+1$, sendo 0 quando se verifica inexistência de autocorrelação (não há efeito da distância sobre a distribuição da variável). Um valor positivo implica agrupamento e um negativo, dispersão. $O$ valor esperado de I é $-1 /(\mathrm{N}-1)$, que se aproxima de 0 , à medida que $\mathrm{N}$ aumenta. $\mathrm{O}$ valor de $\mathrm{z}$ pode ser calculado da maneira convencional, utilizando-se o desvio padrão de I, ou seja, $\sigma$.

Embora seja capaz de apontar a tendência geral de agrupamento dos dados, o I de Moran é uma medida global e, por isso, não revela padrões locais de associação espacial. Para verificar isto, foram calculados os indicadores locais de associação espacial: LISA ("local indicators of spatial association") ${ }^{(9)}$. Estes indicadores testam a hipótese nula de distribuição aleatória por comparar os valores de cada localização específica com os valores de seus vizinhos.

Nesse estudo, a estrutura de vizinhança foi estabelecida por meio de matrizes de pesos espaciais de contiguidade binária do tipo Rook. Os coeficientes de autocorrelação foram considerados significativos quando $p<0,05$. Quando o evento era referente à taxa (proporção), foram aplicados os testes de Moran com Estimativa Bayesiana Empírica para taxas ${ }^{(9-11)}$. Quando o número de pessoas expostas é pequeno, qualquer leve alteração no número de eventos, mesmo que ao acaso, provoca grandes variações no valor da taxa. Marshall(11) propôs a aplicação de Estimadores Bayesianos Empíricos como alternativa ao uso de taxas brutas nessas condições. Esta estimativa computa a média ponderada entre a taxa bruta para cada setor e a média de uma janela espacial, com pesos proporcionais à população em risco, resultando num mapa suavizado.

O indicador Moran Local avalia a significância dos agrupamentos espaciais locais, conhecidos como "hot spots", ao redor de uma localização individual e indica focos de não-estacionaridade espacial (localizações atípicas).

Quatro padrões podem ser visualizados no mapa de associação local:

1. Padrão Alto-Alto (AA): revela setores com alto valor da variável sob análise, cercados de vizinhos com valores semelhantes; 
2. Padrão Baixo-Baixo (BB): setores com baixo valor e vizinhos com valores também baixos;

3. Padrão Alto-Baixo (AB): setores de alto valor e vizinhos com baixo valor;

4. Padrão Baixo-Alto (BA): setores de baixo valor e vizinhos com alto valor.

Os padrões AA e BB revelam associação espacial positiva, enquanto que $\mathrm{AB}$ e $\mathrm{BA}$ demonstram associação espacial negativa.

Todas as medidas para análise da estrutura espacial dos eventos foram calculadas pelo programa Geoda ${ }^{(9)}$.

O protocolo da pesquisa foi aprovado pelo Comitê de Ética em Pesquisa e todos os responsáveis/participantes foram informados sobre os procedimentos, manifestando seu consentimento por escrito.

\section{RESULTADOS}

\section{QUANTO À PREVALÊNCIA DE TRACOMA}

Constatou-se que, em todas as escolas, havia portadores de tracoma. Detectaram-se 79 crianças com tracoma folicular. Não houve casos das outras formas de tracoma.

A taxa de detecção foi semelhante nas quatro séries avaliadas, não havendo diferença significativa na presença de tracoma com relação ao ano escolar. Avaliando-se as diferentes idades, $85,3 \%$ das crianças possuíam entre 7-10 anos. Com relação ao sexo, a distribuição foi semelhante (56,8\%: sexo masculino).

A prevalência de tracoma para os escolares de Botucatu/SP foi de 2,9\%, com taxa de detecção variável nas diversas escolas (de 1,2\% até 17,3\% - Figuras 1 e 2). Entretanto, considerando-se o local de moradia, não foram detectadas regiões com concentração significativa de casos, como demonstrado pelo estudo da distribuição espacial da doença no município (Figura 3).

\section{Quanto À AVALIAÇÃo dA PRESENÇA do tracoma EM RELAÇÃo À DISTRIBUIÇÃO ESPACIAL}

Botucatu pode ser considerada uma cidade de porte médio. Apresenta estrutura espacial socioeconômica similar a da maioria dos municípios brasileiros. Seu sítio urbano alinha-se no eixo longitudinal, com setores de baixa vulnerabilidade social no seu centro, com crescente vulnerabilidade em direção à periferia, de acordo com o IPVS

A análise exploratória espacial (Figuras 1 e 2) permite observar que qualquer que seja a unidade de agregação dos dados (setores de demanda escolar ou polígonos de Thiessen), a prevalência de tracoma não mostra um padrão espacial global, embora ocorra maior prevalência no centro da cidade e porções norte e sul. Tal observação é confirmada pelos resultados da análise espacial. O padrão da prevalência de tracoma de acordo com os setores de demanda escolar não permite rejeitar a hipótese nula de aleatoriedade $(I=-0,45 ; \mathrm{p}>0,05)$. Também os indicadores locais não revelaram setores de demanda significativos. Quando a análise foi feita para os polígonos de Thiessen, o padrão global foi aleatório $(=-0,07$; $p=0,49$ ), mas os indicadores locais apontaram um agrupamento do tipo BB para um polígono ao norte, correspondente à EMEFEl Prof. Luiz Carlos Aranha Pacheco (Figura 3).

A diferença dos resultados pode ser explicada pela diferente agregação das escolas. A análise feita levando-se em conta os setores de demanda escolar não permite separar as escolas como unidades de área, diluindo a prevalência de tracoma nos setores que incluem mais de uma escola. Embora o setor 10 apresente prevalência bem acima da média (Figura 1), não corresponde a um conglomerado alto, de acordo com a análise estatística. A segunda análise, considerando-se os polígonos de Thiessen, permite identificar uma área (setor 14) onde a prevalência de tracoma é menor do que em qualquer outra (Figura 3).

Embora o padrão global da doença não reproduza as condições socioeconômicas da população, a prevalência mais baixa do tracoma é encontrada em setores de menor vulnerabilidade social.

\section{DISCUSSÃO}

Realizou-se um estudo transversal, com a intenção de se detectar os portadores de tracoma inflamatório no município de Botucatu/SP, assim como localizar os casos positivos utilizando o geoprocessamento.

O tamanho da amostra foi estimado em 2.092 crianças, considerando-se a prevalência histórica de tracoma para o município, com erro de estimação de $10 \%$ e nível de confiança de 95\%. Como perdas poderiam ocorrer, a amostra foi acrescida de 20\%. Entretanto, o número de crianças examinadas foi de 2.692 crianças. Isto porque consideramos a classe como a unidade primária de amostra. Havíamos estimado que o número médio de crianças por sala de aula seria de 30 indivíduos, porém, em algumas classes, naquela época, havia mais alunos que o número médio estimado, tendo sido todos os alunos das classes examinados, o que gerou número superior de crianças ao previamente determinado como suficiente. Realçamos que este é o procedimento padrão para estudos de tracoma.

Algumas limitações se impuseram no presente estudo, começando pelo fato de se ter uma prevalência histórica que foi estimada há 10 anos do estudo atual, no qual se apontava que $11,9 \%$ das crianças que frequentavam as escolas apresentavam tracoma naquela época ${ }^{(7)}$. Aquelas crianças foram tratadas, assim como seus comunicantes e, depois daquele estudo, nada mais foi realizado no município. Apesar de o tracoma ser doença de notificação compulsória e de não se ter notícia de casos novos nestes dez anos, muito provavelmente alguns casos foram detectados e tratados, com subnotificação da doença.

Outra dificuldade foi a necessidade de se ter o termo de consentimento assinado pelo pai ou responsável. Houve demora do início da atividade por este motivo, fazendo com que nos aproximássemos das férias escolares. Quando o estudo envolve escolares, as férias devem ser programadas dentro do cronograma, a fim de atingir a população de estudo dentro do tempo previsto.

Em seguida ao estudo realizado em Botucatu no ano de 1995, houve a implantação de um programa de controle de tracoma com tratamento dos casos, controle de comunicantes e educação em saúde. Naquela época, o tratamento foi feito utilizando-se pomada de tetraciclina tópica. Mais recentemente, azitromicina via oral tem sido preconizada pela OMS e pelo Ministério da Saúde brasileiro, pois possibilita maior aderência ao tratamento e melhor controle de administração(12).

Vale ressaltar que, no estudo anterior, a prevalência detectada esteve acima de 10\%, não tendo sido implantado o tratamento em massa, porque esta conduta foi instituída pela OMS apenas no ano de 2003.

Depois daquela primeira pesquisa(7), nenhuma outra intervenção foi feita em Botucatu/SP com relação à pesquisa/tratamento do tracoma. Entretanto, comparando-se aqueles índices $(11,9 \%)$, com os atuais $(2,9 \%)$, houve redução significativa no número de casos no município. A prevalência do estudo atual foi semelhante ao encontrado entre os escolares e préescolares de São Paulo/SP (2,2\%), em 1999(8). Esta prevalência não é elevada, quando comparada a Joinville/SC (4,9\%), Duque de Caxias/RJ (8,8\%) $)^{(13-14)}$, Guaraci/SP $(9,6 \%)^{(15)}$, Chapada do Araripe/PE $(20,2 \%)^{(2)}$ e São Gabriel da Cachoeira/AM (28,02\%)(1). Assim, a redução do número de casos poderia ser resultado do tratamento realizado após a pesquisa anterior. 
Segundo a literatura, quanto menor a endemicidade do tracoma, mais elevada será a idade em que aparecerá o pico de prevalência do tracoma folicular, denotando, assim, uma forma branda da doença ${ }^{(2)}$. Além disso, a idade de início da infecção influencia na evolução, sendo mais grave, quanto mais precoce for seu início(2). As crianças pré-escolares/escolares constituem importantes fontes de infecção ativa ${ }^{(8)}$.

Embora a prevalência encontrada tenha sido baixa e os casos fossem da forma branda da doença (tracoma folicular), caso estes não fossem adequadamente diagnosticados e tratados, o contágio poderia elevar a prevalência ou poderiam os casos evoluir para a forma cicatricial, com lesões incapacitantes. Assim, a existência de crianças com formas de infecção ativa, mesmo com baixa prevalência, constitui fonte de infecção e possibilita a manutenção da cadeia de transmissão do tracoma ${ }^{(8)}$.

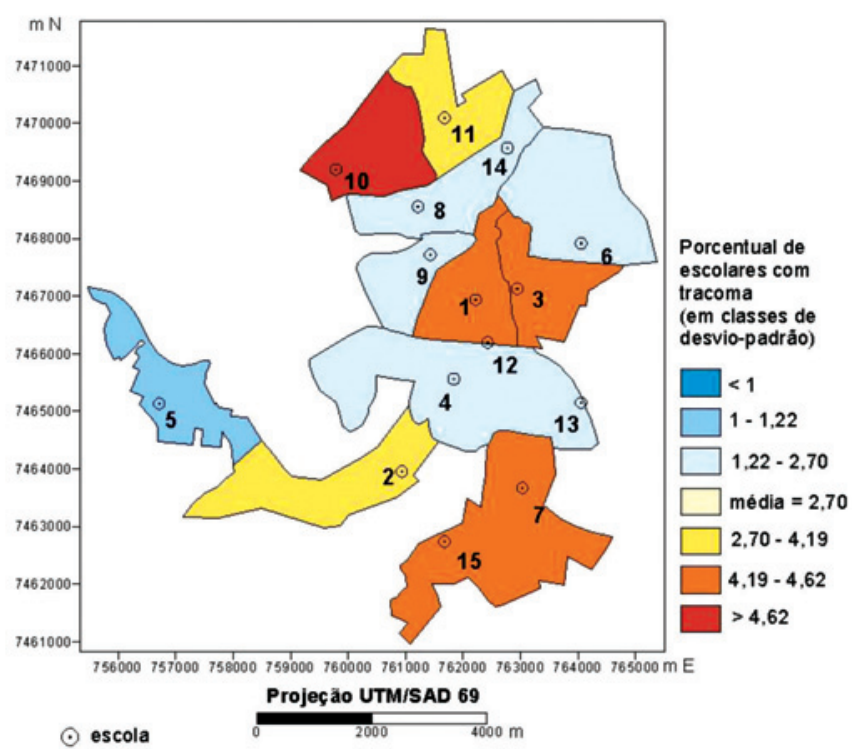

Figura 1. Porcentual de escolares com tracoma, por setor de demanda escolar.

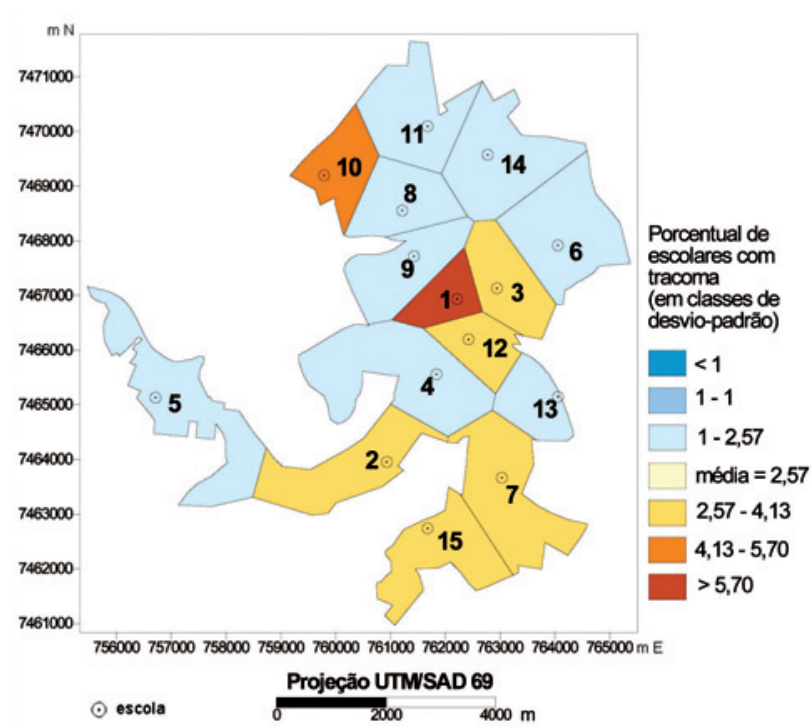

Figura 2. Porcentual de escolares com tracoma, por polígonos de Thiessen.
Entretanto, para uma doença que depende muito de fatores relacionados ao índice de desenvolvimento humano, a queda da prevalência do tracoma em Botucatu/SP poderia ser reflexo da melhoria das condições de saúde da população, visto que a suscetibilidade ao tracoma é geral, ocorrendo com maior frequência onde há falta de água e condições habitacionais/sanitárias inadequadas. Por este motivo, esta doença é um dos indicadores de subdesenvolvimento(8).

Apesar das periferias das cidades possuírem, geralmente, setores de baixa vulnerabilidade social, especificamente para Botucatu/SP, o que se observam, nas periferias, são casas populares e condomínios onde residem as pessoas de maior poder econômico. Não se identificam "favelas" e praticamente todos os domicílios recebem água tratada. Quanto à condição de saneamento básico, nas periferias, há áreas desassistidas do tratamento de esgoto. Porém, na maioria destes locais, o domicílio apresenta fossa séptica. Ademais, Botucatu/SP é um local de nascentes de rios e o abastecimento de água é realizado através destes. Portanto, os fatores ambientais são favoráveis à saúde.

Há áreas com menor desenvolvimento social em algumas regiões da periferia da cidade. Porém, não houve superposição destes indicadores com a ocorrência de tracoma, na faixa etária pesquisada. Assim, as características epidemiológicas do tracoma, neste estudo, não podem ser explicadas pelos indicadores sociodemográficos considerados.

Ressaltamos que as características físicas favoráveis do município de Botucatu/SP ficaram evidenciadas pelo estudo do geoprocessamento, utilizado para a localização espacial dos casos de tracoma detectados nas escolas. O geoprocessamento apontou os locais de moradia, permitindo conhecer a realidade de onde residem os portadores de tracoma, não tendo sido evidenciado nenhum bolsão da doença. Pelo contrário, apontou para áreas mais favorecidas.

O geoprocessamento é uma ferramenta importante para o estudo de doenças do tipo do tracoma, que podem possuir sua gênese relacionada a fatores ambientais. Os autores acreditam que este tipo de avaliação deva ser cada vez mais utilizada para planejamento em saúde.

Além das condições favoráveis em termos de disponibilidade de água e tratamento do esgoto, o município de Botuca-

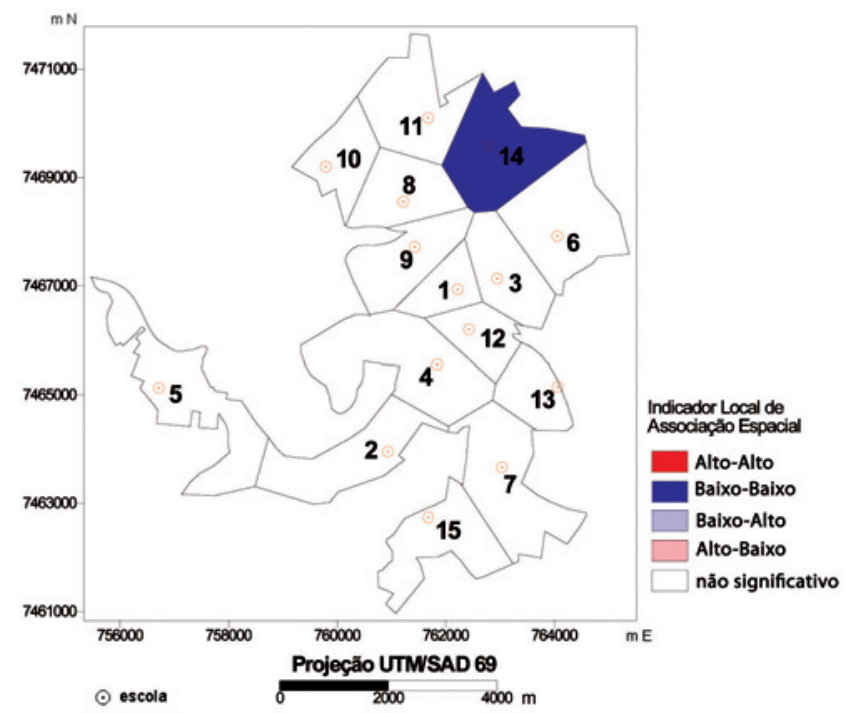

Figura 3. Mapa dos indicadores locais de associação espacial para os polígonos de Thiessen. 
tu/SP tem ainda outros facilitadores. O município dispõe de serviços médicos gratuitos em larga escala. A presença do centro universitário da UNESP garante a possibilidade de detecção precoce e tratamento das afecções, antes que se instalem consequências mais graves.

A busca ativa nas escolas e realização sistemática de investigação epidemiológica de todos os casos detectados é de extrema importância, devido a alguns fatores: falta de sintomas patognomônicos do tracoma, faixa etária mais acometida e elevada taxa de prevalência entre os comunicantes domiciliares ${ }^{(8)}$.

Não obstante à sua aparência clínica branda na infância, o tracoma pode progredir por muitos anos e o comprometimento da visão ocorrer após a meia-idade. Assim, mediante ao conhecimento da patogênese do tracoma, não se deve esperar que os pacientes procurem espontaneamente o tratamento, mas sim isto deve servir de estímulo para a realização de novas pesquisas de campo.

É mister que a equipe conheça a fundo o caminho a ser seguido quando se pesquisa tracoma, já que a detecção implica notificação, tratamento e busca de comunicantes, ou seja, a detecção acaba por ser o mais simples dentro do que é necessário realizar quando se pretende eliminar a cegueira decorrente do tracoma. Estabelecer o tratamento do portador e dos comunicantes, assim como a realização dos controles de cura, deve ser a meta final do pesquisador.

\section{CONCLUSÃO}

A prevalência de tracoma em escolares de Botucatu no ano de 2005 foi de 2,9\%. A análise da distribuição espacial dos casos detectados não revelou áreas de maior vulnerabilidade. Os autores realçam o uso do geoprocessamento como ferramenta para detecção espacial dos portadores, o que pode colaborar para a determinação de medidas para o controle da doença.

\section{AGRADECIMENTOS}

Os autores agradecem a assessoria feita pela Dra. Lígia
Barrozo Simões no tocante à realização do estudo de distribuição espacial.

\section{REFERÊNCIAS}

1. Garrido C, Guidugli T, Campos M. Estudo clínico-laboratorial do tracoma em população indígena da Amazônia Brasileira. Arq Bras Oftalmol. 1999;62(2):132-8.

2. Lucena AR, Cruz AAV, Cavalcanti R. Estudo epidemiológico do tracoma em comunidade da Chapada do Araripe - Pernambuco - Brasil. Arq Bras Oftalmol. 2004 67(2):197-200.

3. Adan CBD, Scarpi MJ, Guidugli T. Eficácia da ciprofloxacina e da tetraciclina no tratamento do tracoma: estudo clínico e microbiológico. Arq Bras Oftalmol. 1996; 59(6):592-600.

4. Pelicioli MCF, Pelicioli AC, Medina NH, Massaini MG. A educação em saúde na prevenção, tratamento e controle do tracoma em uma creche do município de São Paulo [relato de experiência]. Rev Bras Saúde Esc= Braz J Sch Health. 1992;2(3/4): $178-85$.

5. Luna EJA, Medina NH, Oliveira MB. Vigilância epidemiológica do tracoma no Estado de São Paulo. Arq Bras Oftalmol. 1987:50(2):70-9.

6. Carvalho RC, Falcão R, Cohen J, Chaves C, Scarpi MJ. Prevalência de tracoma em escolares de Manaus. Arq Bras Oftalmol. 1997;60(3):243-7.

7. Medina NH, Gattás VL, Anjos GL, Montuori C, Gentil RM. Prevalência de tracoma em pré-escolares e escolares no Município de Botucatu, São Paulo, Brasil, 1992. Cad Saúde Pública = Rep Public Health. 2002;18(6):1537-41.

8. Koizumi IK, Medina NH, D'Amaral RKK, Morimoto WTM, Caligaris LSA, Chinen N, et al. Prevalência do tracoma em pré-escolares e escolares no município de São Paulo. Rev Saúde Pública = J Public Health. 2005;39(6):937-42.

9. Anselin L. Local indicators of spatial association - LISA. Geogr Anal. 1995;27(2):93-115.

10. Assunção RM, Reis EA. A new proposal to adjust Moran's I for population density. Stat Med. 1999;18(16):2147-62.

11. Marshall RJ. Mapping disease and mortality rates using empirical Bayes estimators. J R Stat Soc Ser C Appl Stat. 1991;40(2):283-94.

12. São Paulo (Estado). Secretaria de Estado da Saúde. Centro de Vigilância Epidemiológica Professor Alexandre Vranjac. Manual de vigilância epidemiológica: tracoma - normas e instruções. $2^{\underline{a}}$ ed rev. São Paulo: Centro de Vigilância Epidemiológica;1993.

13. Couto Júnior AS, Scarpi MJ, Guidugli T. Prevalência de tracoma em pré-escolares e escolares no Município de Duque de Caxias - RJ. Rev Bras Oftalmol. 1997:56(7):515-21.

14. Nóbrega MJ, Farah ALHL, Miller D, Kang HM, Haddad LH. Estudo clínico e laboratorial do tracoma em escolares de Joinville, Santa Catarina, Brasil. Arq Bras Oftalmol. 1998;61(5):551-6.

15. Medina NH, Oliveira MB, Tobin S, Kiil G Jr, Mendoca MM, de Barros OM, et al. The prevalence of trachoma in preschool and school children in Olimpia, Guaraci and Cajobi, São Paulo, Brazil. Trop Med Parasitol. 1992:43(2):121-3.

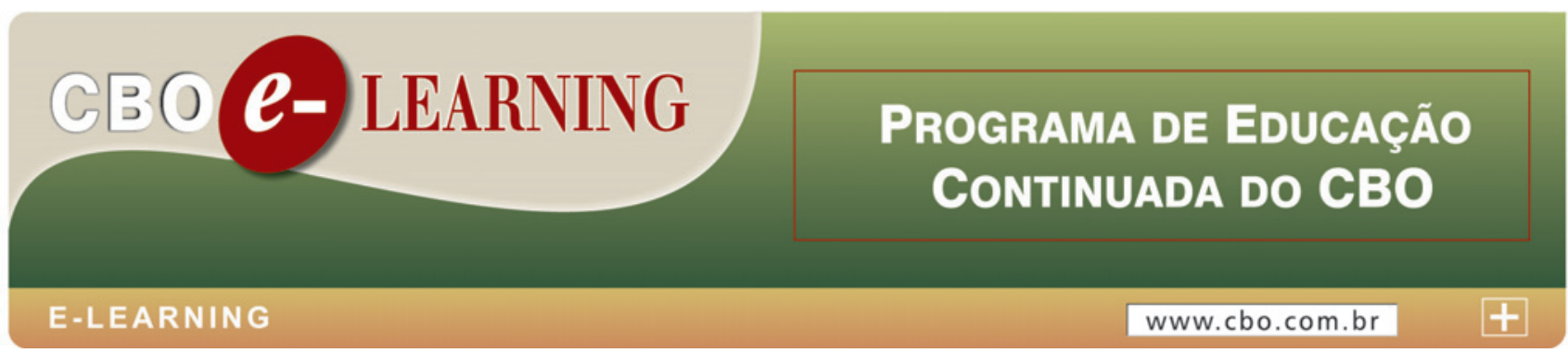

